\title{
THE CYCLIC EVOLUTION OF CLASSICAL NOVAE
}

\author{
Dina Prialnik \\ Department of Geophysics and Planetary Sciences \\ Raymond and Beverly Sackler Faculty of Exact Sciences \\ Tel Aviv University, Ramat Aviv 69978, Israel
}

\section{Introduction}

The cyclic evolution of classical novae (CN) cannot be observed, as in the case of recurrent or dwarf novae. The duration of a typical cycle may range from a few thousand to a few $10^{5}$ years. Thus we have to rely on theoretical studies for understanding the periodic outbursts - due to thermonuclear runaways (TNR) - on the surface of accreting white dwarfs (WD). One may distinguish between two kinds of studies: a) detailed investigations of a specific aspect or a particular evolutionary phase; b) studies which emphasize the cyclic behavior of novae and their long-term evolution. The latter, which form the subject of this brief review, may be divided into four groups, each adopting a different approach to the problem:

1. Steady-state (semi-analytic) solutions;

2. One- (or two-) zone models;

3. Quasi-static (and/or steady burning) numerical calculations;

4. Full-scale hydrodynamic evolutionary computations.

The evolution of a CN involves periodic changes in the structure of the outer layers of an accreting WD and is therefore described by a series of functions of time and space (usually one-dimensional, the distance from the surface of the WD core). Models in the first group focus on the space dependence of these functions, by seeking steady-state solutions. Those in the second group focus on the time dependence of the characteristic functions, by using average integrated quantities. In the third group of models the evolution in time is approximated by a series of static models. Finally, in the full-scale models the complete set of evolutionary equations is solved, allowing for the development of convection, dynamic processes and nucleosynthesis. However, the lengthy calculations involved are thus limitted to one or two full cycles. Only by combining the results and conclusions of all the types of models, we may hope to understand the long term, cyclic evolution of $\mathrm{CN}$ and the variety of characteristics that they exhibit.

\section{Results of analytical models}

Analytic solutions for the structure of the envelope around a WD (Fujimoto, $1982 \mathrm{a}, \mathrm{b})$ show that for a given $\mathrm{WD}$ mass and envelope mass there are two distinct types of stable configurations in thermal equilibrium: a 'high' state and a 'low' state, characterized by a high and a low entropy value at the envelope base, respectively. In the high state the nuclear shell burning supplies the energy lost at the surface. There exists a lower limit to the envelope mass for this state. In the low state nuclear burning 
is extinct and thermal balance is achieved by compressional heating, which compensates for diffusion and radiation of heat. There is an upper limit to the envelope mass of this state, set by the ignition of nuclear reactions when the mass has exceeded a critical value. An accreting WD oscillates between these sfates, as accretion drives the envelope mass beyond the upper limit of the low state, whereas nuclear burning and mass loss reduce it past the lower limit of the high state. Thus a cyclic evolutionary pattern emerges, even when stable, stationary solutions are sought.

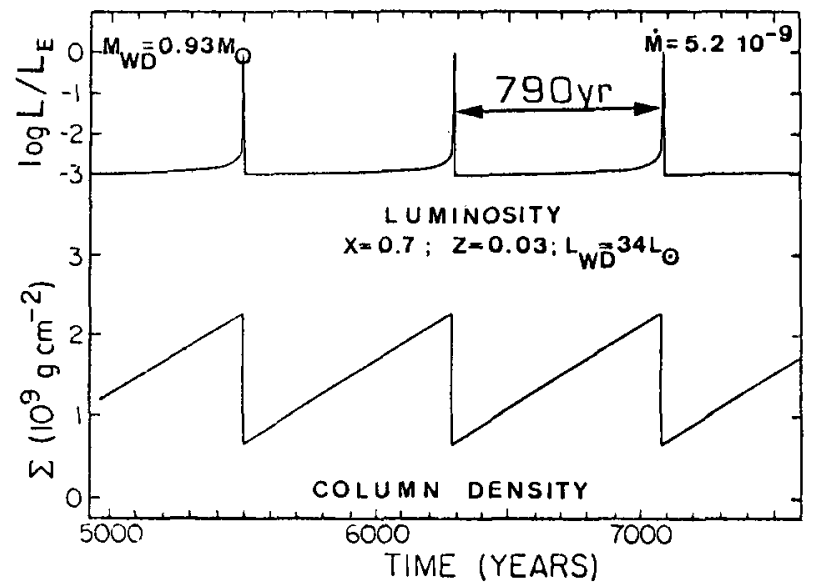

FIGURE 1

Estimates of the cycle's duration and the schematic time-dependent behavior are obtained by the second approach. Here the entire hydrogen-rich envelope is treated as one mass zone (Paczynski, 1983) - or, sometimes, two zones (e.g. Buchler and Perdang, 1979) - overlying a core of fixed size. In the stellar structure equations all functions under integrals are replaced by their values at the top or the bottom of the shell. The result is a pair of ordinary differential equations, which can be readily solved for a given opacity law, nuclear energy generation rate and equation of state, assuming radiative equilibrium. Three parameters have to be specified: the accretion rate, the gravitational acceleration at the WD surface (i.e. the WD mass) and the heat flux at the bottom of the envelope (i.e. the WD luminosity or temperature). These, in fact, constitute the basic parameters of a nova event. An example of the results obtained is given in Fig. 1. The main conclusions reached by this procedure are:

1. Nuclear burning proceeds in short bursts (flashes) separated by long periods of no burning. The quiescent periods are roughly inversely proportional to the accretion rate and decrease with increasing heat flux from the core.

2. The instability develops very quickly and during the flash $L \approx L_{E d d}$.

3. The instability is suppressed (and the cyclic evolution disappears) when the WD is very hot, when the accretion rate is either very high or very low, or for vanishing metal content in the envelope, when the only source of nuclear energy is the $p$-p chain reaction. 
4. Usually, a full amplitude and a constant time interval between flashes are reached within two or three flash cycles.

It is remarkable that all the outstanding characteristics of the cyclic evolution of novae are obtained by relatively simple analyses.

\section{Results of numerical calculations}

The dynamic phase of a nova cycle is of relatively short duration: it includes the rapid expansion following the thermonuclear runaway and the ensuing period of mass ejection. Therefore one is justified in treating most of a cycle by means of the quasistatic approximation. Such calculations (e.g. Paczynski and Zytkow, 1978; Iben, 1982; Papaloizou, Pringle and MacDonald, 1982; Sion and Starrfield, 1986) yield more accurate descriptions of the nova cycle, as shown in Fig. 2 (adapted from Iben, 1982). The outburst itself is followed under the assumption of thermal and hydrostatic equilibrium. The resulting dependence of the nova cycle outburst and (in italics) quiescent periods on basic parameters - the WD mass and the accretion rate - is given in Table 1.

TABLE 1: OUTBURST AND QUIESCENT PERIODS (YEARS)

RESULTING FROM QUASI-STATIC CALCULATIONS

FOR WD MASSES RANGING FROM $0.8 M_{\odot}$ TO $1.4 M_{\odot}$

\begin{tabular}{|l|l|l|l|l|}
\hline $\log \dot{M}$ & 0.8 & 1.0 & 1.2 & 1.4 \\
\hline-11 & 2800 & 220 & 21 & 2.2 \\
& $5.1(7)$ & $6.8(6)$ & $9.1(5)$ & $1.2(5)$ \\
-10 & 1300 & 110 & 10 & 1.0 \\
& $2.5(6)$ & $3.3(5)$ & $4.4(4)$ & 5800 \\
-9 & 620 & 49 & 4.7 & 0.48 \\
& $1.1(5)$ & $1.5(4)$ & 2000 & 270 \\
-8 & 270 & 21 & 2.0 & 0.20 \\
& 4800 & 630 & 84 & 1.1 \\
-7 & 120 & 18 & 1.5 & 0.15 \\
& 280 & 38 & 5.0 & 0.66 \\
\hline
\end{tabular}

Only a detailed hydrodynamic calculation can supply the characteristics of a nova outburst that may be directly compared with observations (the luminosity, radius and effective temperature of the WD, the velocity of expansion and composition of the ejecta, the rate and duration of mass loss and the rate of decline). Moreover, in order to remove the arbitrariness of initial conditions, more than one cycle is to be computed. Such a calculation (Prialnik, 1986) for a $1.25 M_{\odot}$, accreting at a rate of $10^{-11} M_{\odot} y r^{-1}$ yields the results shown in Fig. 3 for the change with time of the luminosity - bolometric and nuclear - and of the envelope structure: the growth and drop of the envelope mass (note that $m=0$ at the surface of the original WD and increases outwards), 

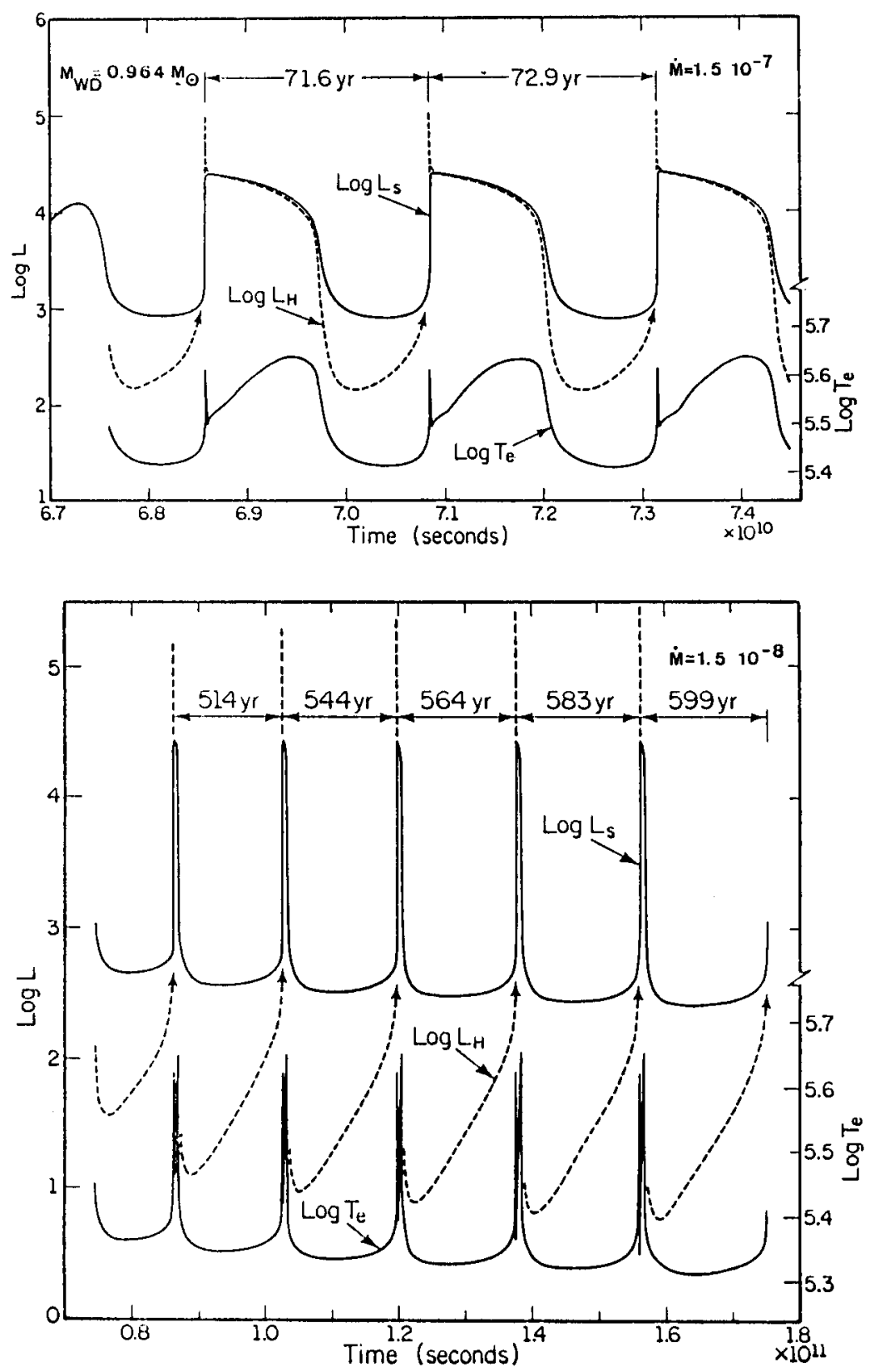

FIGURE 2 

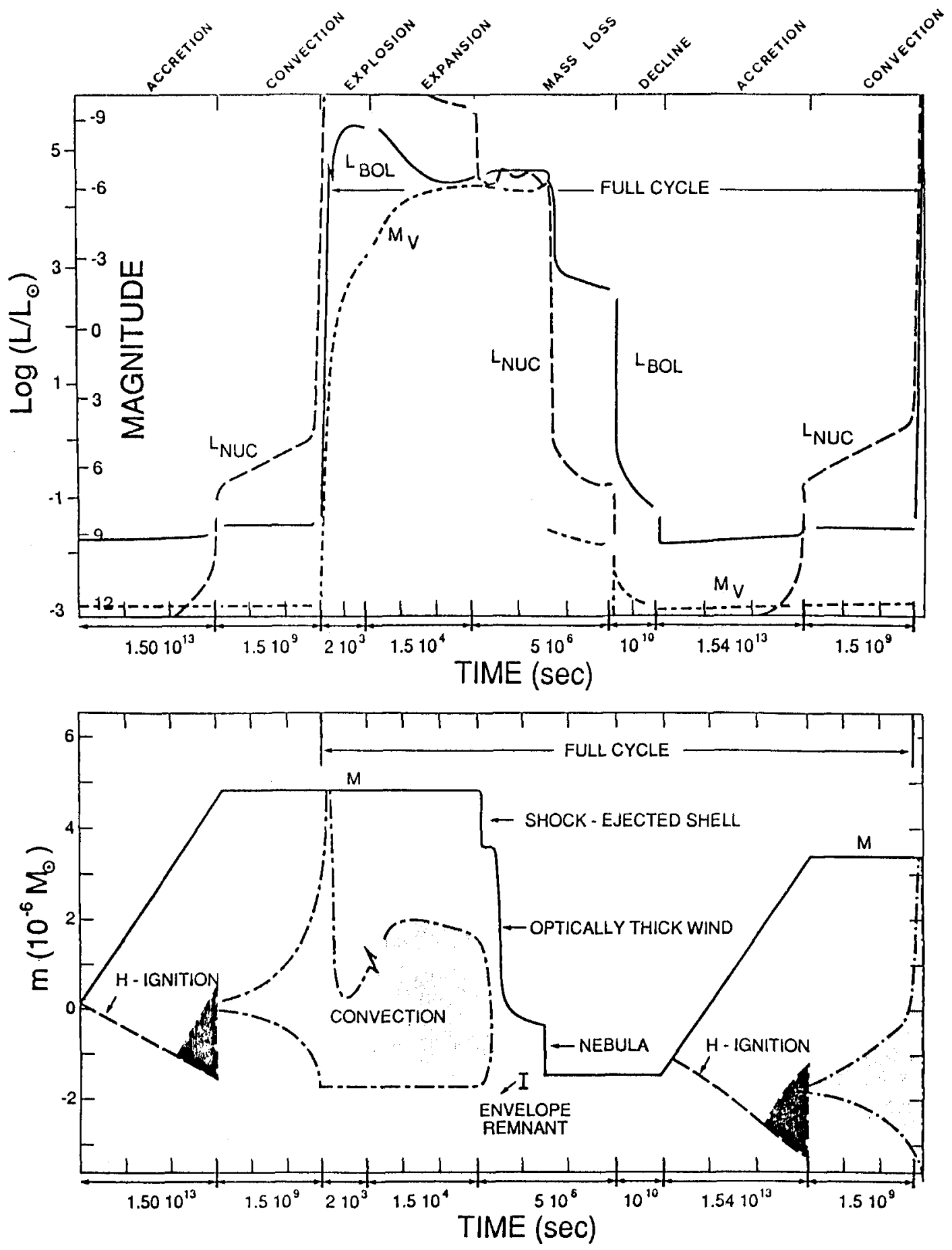

FIGURE 3 
the advance and retreat of convection and the progress of hydrogen burning. A full nova cycle may be divided roughly into six stages: accretion, convective instability, outburst (TNR), followed by rapid expansion, mass loss and, finally, decline. Each stage proceeds on its own characteristic time scale, ränging from a few $10^{5}$ years for the first, down to less than an hour for the TNR - the rise of nuclear luminosity to its peak value. Diffusion (see Prialnik and Kovetz, 1984) during the accretion stage, followed by convection at the onset of the outburst, results in mixing of WD material into the accreted envelope, raising the value of $Z$ in this case to 0.3 . The outburst reproduces the main characteristics of observed fast novae: duration $\left(t_{3} \approx 25\right.$ days $)$, plateau bolometric luminosity $\left(4 \times 10^{4} L_{\odot}\right)$, ejected mass $\left(6.5 \times 10^{-6} M_{\odot}\right)$, velocities (ranging from 3800 $\mathrm{km} / \mathrm{sec}$ down to a few tens $\mathrm{km} / \mathrm{sec}$ ). The composition of the ejected mass is in excellent agreement with the observed composition of novae with similar Z (e.g. Nova Cyg 1975 or Nova Cyg 1978). The return to a state typical of novae in quiescence occurs within $10 \mathrm{yr}$ after outburst. The nova remnant continues to cool for a few hundred years, when it regains its preoutburst structure. The evolutionary track in the $L-T_{e}$ diagram is shown in Fig. 4.

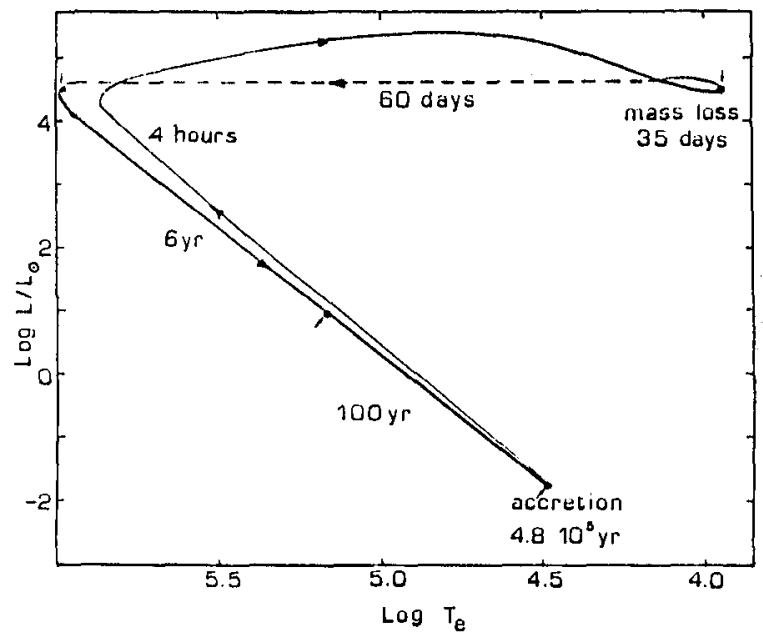

FIGURE 4

The following outburst, after another period of accretion, is found to be very similar to the first. The influence of the thermonuclear runaway and its consequences on the nova progenitor is transient; the WD has ample time to recover during the long quiescent phases that separate eruptions. In fact, it is found (Prialnik, 1987) that the occurence of outbursts at the surface does not interfere with the normal cooling of the WD. (One should bear in mind, though, that this conclusion is based on the calculation of only two consecutuve outbursts.)

Another example of full-scale hydrodynamic calculation (Livio, Prialnik and Regev, 1989 ) is that of two full cycles in the evolution of a $1 M_{\odot}$ WD of a relatively high luminosity $\left(L_{W D}=7 L_{\odot}\right)$, accreting at a high rate of $10^{-8} M_{\odot} y r^{-1}$. The resulting characteristics of this model, as shown in Fig. 5, are very similar to those of the slowest 

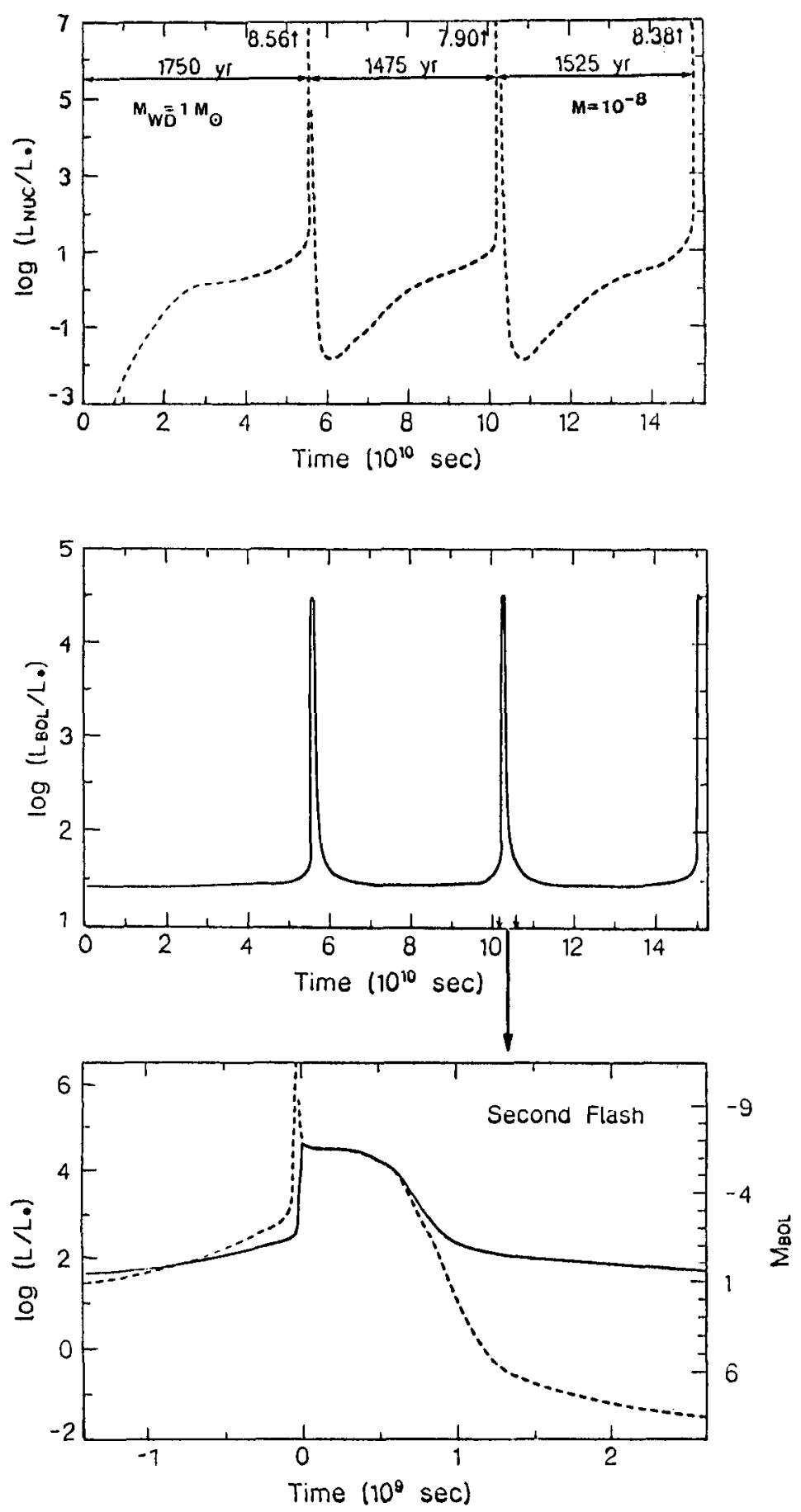

FIGURE 5 
novae (classified also as symbiotic novae) $R R$ Tel and $R T$ Ser. The duration of the quiescent accretion phase is $1500 \mathrm{yr}$, the rise time is of the order of $1 \mathrm{yr}$, and the high, plateau luminosity $\left(L \approx L_{E d d}\right.$ ) phase lasts for about $15 \mathrm{yr}$. The outburst amplitude is of about $8^{m}$ and in the course of the flash roughly half of the hydrogen-rich envelope $\left(8 \times 10^{-6} M_{\odot}\right)$ is ejected as a $10^{-6} M_{\odot} y r^{-1}$ wind, with velocities that do not exceed 40 $\mathrm{km} / \mathrm{sec}$. This, however, should be regarded as a lower limit to the ejected mass (see Kato and Hachisu, 1988).

We have shown examples of very fast and very slow nova models, obtained for different combinations of the three basic parameters: $M_{W D}, L_{W D}$ and $\dot{M}$. By choosing other combinations one should be able to account for the entire range of nova characteristics. Then the question will remain whether a given nova system may go, in the course of its evolution, through the entire range of outbursts.

\section{Repeated cycles and long-term evolution}

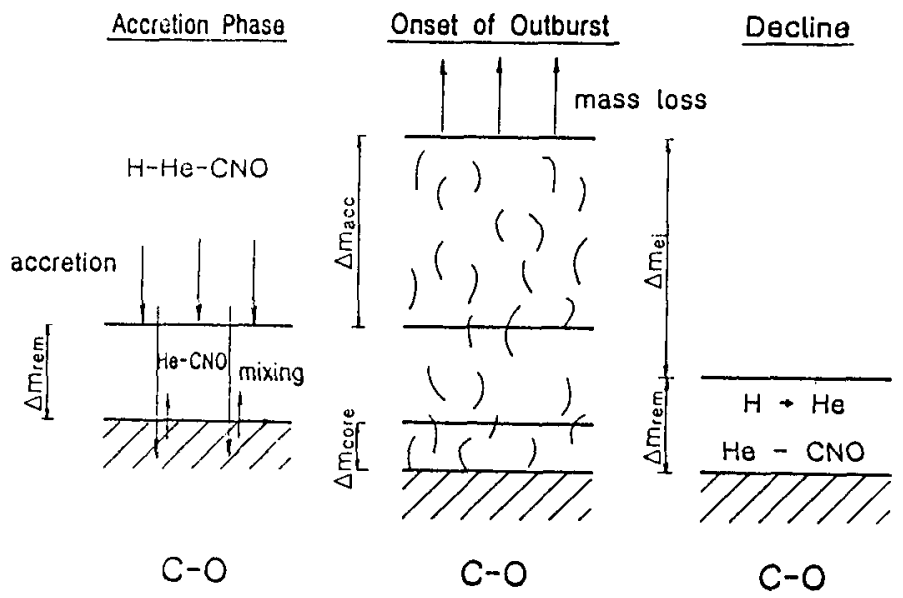

FIGURE 6

The change in structure of the nova envelope during a full cycle is shown schematically in Fig. 6. The accreted mass accumulates on top of a relatively thin mantle ccmposed of helium, produced at the end of the previous outburst, and heavy elements dredged up from the core at the onset of that outburst. Diffusive (or other) mixing occurs through this layer down to the WD core. The deepest point reached by downward diffusing hydrogen in an amount sufficient for ignition defines the base of the new envelope, which becomes fully convective at the onset of the runaway. The envelope is then enriched in CNO elements, mainly from the core, and in helium from the remnant. Various degrees of enrichment may be obtained, according to the relative contribution of core mass $\left(\Delta m_{\text {core }}\right)$, remnant mass $\left(\Delta m_{\text {rem }}\right)$ and accreted matter $\left(\Delta m_{a c c}\right)$ to the final envelope $\left(M_{e n v}=\Delta m_{a c c}+\Delta m_{\text {rem }}+\Delta m_{\text {core }}\right)$. The global characteristics of a cycle depend upon the three parameters already mentioned. These change very slowly 
with repeated outbursts. Otherwise, consecutive outbursts are shown by computations to be extremely similar. We can make use of this feature in the following way: we may define two parameters $\alpha=\Delta m_{a c c} / M_{\text {env }}$ and $\beta=\Delta m_{r e m} / M_{e n v}$ which, along with the envelope composition, would change imperceptively from one outburst to the next. For well observed novae $\alpha$ and $\beta$ may then be derived from the composition $(X, Y, Z)$ of the ejected envelope, assuming solar abundances $\left(X_{\odot}, Y_{\odot}, Z_{\odot}\right)$ for the accreted matter and $(0,1-Z, Z)$ and $(0,0,1)$ for the hydrogen, helium and heavy element mass fractions in the remnant and in the core, respectively. Thus,

$$
X=\alpha X_{\odot} ; \quad Y=\alpha Y_{\odot}+\beta(1-Z)
$$

lead to

$$
\alpha=X / X_{\odot} ; \quad \beta=\frac{Y / X-(Y / X)_{\odot}}{1+Y / X}
$$

TABLE 2: OBSERVED ABUNDANCES (X, Y, Z) AND RESULTING PARAMETERS

\begin{tabular}{|ll|l|l|l|l|l|}
\hline \multicolumn{2}{|c|}{ Nova } & $\mathrm{X}$ & $\mathrm{Y} / \mathrm{X}$ & $\alpha$ & $\beta$ & $\mathrm{Z}$ \\
\hline $\mathrm{RR}$ & Pic & 0.50 & 0.90 & 0.68 & 0.29 & 0.05 \\
$\mathrm{DK}$ & Lac & 0.47 & 1.00 & 0.64 & 0.33 & 0.06 \\
$\mathrm{HR}$ & Del & 0.45 & 1.04 & 0.62 & 0.34 & 0.08 \\
$\mathrm{CP}$ & Lac & 0.60 & 0.43 & 0.82 & 0.06 & 0.14 \\
$\mathrm{~T}$ & Aur & 0.44 & 0.88 & 0.60 & 0.29 & 0.17 \\
V1500 Cyg & 0.49 & 0.43 & 0.67 & 0.06 & 0.30 \\
V1668 & Cyg & 0.47 & 0.47 & 0.64 & 0.09 & 0.31 \\
V693 & CrA & 0.29 & 1.10 & 0.40 & 0.36 & 0.39 \\
DQ & Her & 0.30 & 0.57 & 0.41 & 0.14 & 0.53 \\
V1370 & Aq1 & 0.05 & 1.80 & 0.07 & 0.52 & 0.86 \\
\hline
\end{tabular}

The results of this exercise are given in Table 2 . It is interesting to note that there is no correlation between the heavy element content of the ejecta and the fraction of the envelope mass that was ejected, although one should have expected that a strong outburst, triggered by high $\mathrm{Z}$ values, would result in extensive mass ejection.

The trend of change of the nova parameters due to the accumulated effect of many outbursts is toward cooler and slightly smaller WD's, and lower accretion rates (resulting from decreasing orbital periods). Each one of these factors alone causes the eruption period to increase. Therefore, any given nova system should evolve into less frequent outbursts and, as indicated by the parameter study of Kovetz and Prialnik (1985) - 


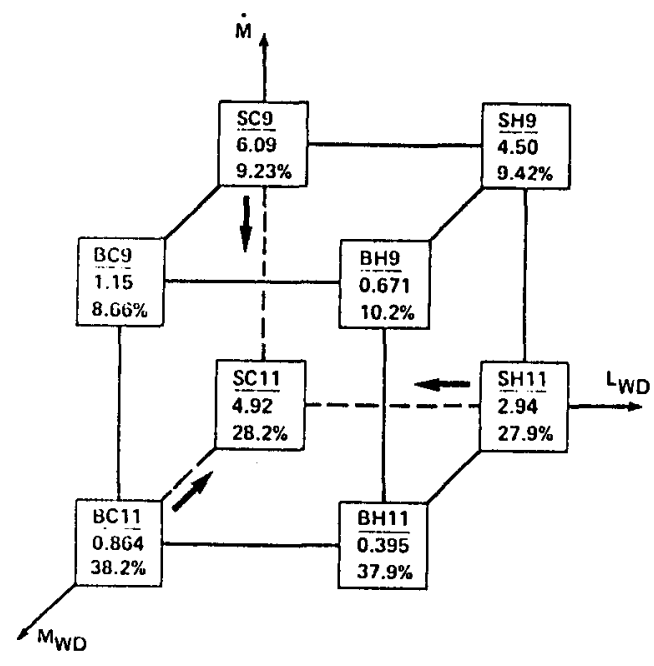

FIGURE 7

summarized in Fig. 7 - higher envelope (and ejected) masses, and probably higher metal content in the ejecta.

\section{References}

Buchler, J.R. and Perdang, J. 1979, Ap. J., 231, 524.

Fujimoto, Y.F. 1982a, Ap. J., 257, 752 .

Fujimoto, Y.F. 1982b, Ap. J., 257, 767.

Iben, I.Jr. 1982, Ap. J., 259, 244.

Kato, M. and Hachisu, I. 1988, Ap. J., 329, 808.

Kovetz, A. and Prialnik, D. 1985, Ap. J., 291, 812.

Livio, M., Prialnik, D. and Regev, O. 1989, Ap. J., 341, 299.

Paczynski, B. 1983, Ap. J., 264, 282.

Paczynski, B. and Zytkow, A.N. 1978, Ap. J., 222, 604.

Papaloizou, J.C.B., Pringle, J.E. and MacDonald, J. 1982, M.N.R.A.S., 198, 215.

Prialnik, D. 1986, Ap. J., 310, 222.

Prialnik, D. 1987, Mem. S.A.It.,58, 139.

Prialnik, D. and Kovetz, A. 1984, Ap. J., 281, 367.

Sion, E.M. and Starrfield, S. 1986, Ap. J., 303, 130. 